\title{
Study on the Alienation of Students Communication Style under the
}

\section{Perspective of Daily Life}

\author{
Kun Jing ${ }^{1}$ \\ ${ }^{1}$ Quzhou College, Quzhou, Zhejiang, 324000
}

KEYWORDS: Daily Life; Students Intercourse; Alienation

\begin{abstract}
Respect daily life and develop education value of daily life for college students in the age of the Internet have a natural effect on overcoming the growing problem of alienation. Students alienation intricate genesis point of view mainly due to lack of education in daily life. Return rational state of daily life is the core strategy to solve this problem. Without the open world of daily life, daily management education of college students will not be able to emerge and cannot cope with all kinds of alienation impact.
\end{abstract}

\section{Introduction}

"Daily Life in the world of daily life and integration of non-World covers virtually all areas of the human world." The first is the everyday person, or he does not exist. Daily life itself along with each person's life has always been, people need to break free from the original daily life indifferent and even despise the state, began to try to study its important value, of which the most important is that for daily life educational value of respect and digging. "Daily life beginning as a basic human growth environment and the people in this environment of growth (life events) have been defined. Therefore, the daily life of the people is the world's first basic education." First of all, the daily life is the source of educational content lies, life is not only of great significance in terms of moral education, it also provides intellectual and practical skills, physical development, and many other creative content. Second, daily life is a process of educating people carrier, the educational process is basically consistent with the echoes of daily life processes. Although daily life is taboo Mo, but it faithfully record the effectiveness of educational play, save from quantitative to qualitative change process, which evaluate the current education and development advocacy coincide. Third, the daily life is the goal of testing and education indicators, and all educational outcomes will eventually be put up to show the stage of life and acceptance of daily life evaluation objective and fair. The purpose of life is life itself, rather than to go to educate others, so as to guide education returning to the highest goal of life, daily life plays a good daily advantage, comprehensive abandon the utilitarian dislocation goal-oriented education.

\section{The Performance of College Students Way of Communication Alienation}

Habermas believes that "alienation means alienation relationship between body, not equality between people, trust, natural interaction, but transformed into a distorted, inequality, distrust and unnatural subject-object relationship. Communicative action is the people's daily life must be among the world's exchanges also reflects the alienation of life in the world of alienation, and only return to a rational world of daily life, with each other rational communication between people in order to become possible. 
After the contemporary "95" has been replaced by "80" has become the main force of the university campus, their daily way of communication there are different degrees of alienation tendencies. MyCOS Institute published a 95 freshmen after survey shows that $45 \%$ of college freshmen are most worried about relationships, after 39\% of the 95 students said the presence of interpersonal problems. Visible, after 95 freshmen relatively more worried about interpersonal issues. There are even refused to associate, extreme cases simply close ourselves up. With their predecessors '80' compared to this alienation is reflected in the first contact practice of daily life among college students, the impact in this area is very clear, which has become the root of a series of subsequent alienation of the problem.

First, exchanges subject alienation. Students launch as interpersonal communication, their self-positioning and self-alienation relationship. Specific performance, Role deformity, all self-centered, arrogant, positioning itself as the University of daily life and interaction absolute master, and all the other exchanges as the main objects and tools. The situation cannot fulfill its role in family life, social life conversion to the role of the environment, therefore, only consider exchanges to maximize self-interest, ignoring the feelings of others, contempt for all the rules and regulations, failing to deal with the extreme lack of rational thought. And by the bad behavior labeled "self-assertive" personalized labels to be rationalized.

Second, communication media alienation, can also be called media rely on technology or media-oriented way of life. This is a typical manifestation of distorted human nature alienation. Smart phones and network technology as the representative of a variety of new media is the result of human activity should be invented, it should exist as an object of human activity, but the reality is that college students suffering from media addiction, lost their subjectivity, any media can become a master of their daily lives, cutting their master active time, turn restrictions, slavery, oppression, people become a tool of such activities, and even slaves. The university classroom "mobile phone overuse" figure uncommon, mobile phones have become the manufacturing estrangement and conflict between teachers and students another tool.

Third, the interaction object alienation, objectification of the body. Students in the communication process, adhere to the guiding utilitarian exchanges as both sides take each other to meet their own needs and desires of some means or tool, it is to ignore the other's reasonable emotional factors, quality, dignity, psychological experience like the other person treated as objects, objects, abstract entities. The other is based on mutual exchange of value for their own use size. "Friends are brought Utilization" is the motto of this group pursued dating. Stress face interaction process, emphasizing the identity matched to one another "perfect match" type, small campus in human society divided into 369 and so on.

Fourth, the degree of interaction and content alienation. Contacts and relations between the surfaces of the perfunctory, college students in the communication process is often lost the desire to peer friendship, become lonely heart dancers. Closed down, with other people just stay in the resort entertainment courtesy and polite hypocrisy to pretend enthusiasm level, especially when the other powerless to be made, such a performance is more obvious, it is a formidable passive mode of communication. Communication content of worships money, by the impact of the global consumer culture, the blind pursuit of contacts in the convergence of consumer content, the performance of the exchanges materialistic, concerned with money, that "money first", uphold the "universal theory of money."

\section{The Analysis of the Causes of Students Alienation}


Macro-social environment of daily life alienation affected societies from daily life of people and things everywhere. According to Marx's theory of alienation perspective, alienation is the alienation between man and man, which is the core of the theory of alienation. In modern society, especially by the market economy changes and the impact of the current relationship between China, as the main body between people from the original equality, trust, between the main body by the laws of nature and impact of interactive state degenerated into a distorted, not to be trusted, unnatural relationship between subject and object. This state of alienation is bound to extend to the university campus in the life of the world, and the problems caused by interaction between the teacher-student relationship alienation Students will be inevitable.

Meso-level, college interpersonal education for college students of insufficient supply, resulting in interpersonal skills college student short board, resulting in alienation from the occurrence of the origin of the body. University campus is still learning at the core of non-dominated rhythm of daily life, people will be more time and energy in daily life or on the non-mainstream content. Daily life has not completely caused by education and managers enough attention, always be seen as a non-accessory of daily life and the presence of a minor. While traditional education only way to re-evaluate the results, we do not care about the process, as a non-daily life learning process can turn a blind eye, that daily life for students during intercourse whether alienation, even more attend the. People do not just appear on the signs of alienation interpersonal, it is not cause enough concern and attention. From the core inside the point of view, this alienation of state exchanges, is the fundamental source is the lack of ability of college students with people, and for this reason is the lack of long-term ability of the lack of culture in this area and the absence of appropriate education and insufficient. Life Education Association Education due to insufficient supply, which is the ultimate attributed to a variety of other incentives.

The level of media contacts, applications and "younger" popularity of "smartphones" as the representative of the diverse new media makes this alienation worse. Behavior and way of thinking after 95 have been closely linked up with the new media, communication and before a lot of people rely on to solve the problem, now have become a communication between man and machine. This media-oriented way of life not only reduces the chance of face to face interaction between college students, but also weakened the language and the ability to interact with them in person. Psychologists worry that a variety of network communication tools has been a serious threat to the normal interviews social way, over time, many people may lose the basic ability to communicate with people Discourse. Such a state of alienation is bound to be more worried about 95 college students after campus interpersonal problems, in their eyes, compared with the virtual world of communication methods, daily interactions in real life is much more complex and frightening.

The micro level, individual student daily life cognitive dislocation. Students 95 after the same is psychological, "weaning", which may be more difficult to adapt than physiological weaning period. Their life cognitive ability is poor, living in the world to understand is not comprehensive enough. High school, due to heavy academic burden and parents acting on their behalf, they are often as their daily lives supporting the existence of, and to the university level, it is bound to face from the original own daily life supporting targeting to daily life principal role and identity transition issues. In university life in the face of everything to re-learn, re-adapt and re-start, interpersonal identity should be repositioned for those willpower is not strong, then students' individual learning ability is poor, there psychologically lost confused, loss, showing stress the extreme alienation of the behavior is inevitable. This is mapped to the inevitable alienation of social life effects of individual student body. 


\section{Play the Educational Function of Daily life}

Daily life has a natural educational exclusion of alienation both exchanges Students daily life education content, but also the specific management education pathways. Life should be a rational world the highest level of college students to overcome the alienation. In which we can develop educational resources from the following aspects.

First, the daily life of a university student exchanges happen carrier. The problems of alienation are in this stage show most vividly. To overcome the alienation of the carrier body have the advantage of proximity to daily life as the goal-oriented, integrated campus resources, so that students learn in the process of living growth. To create a rational and harmonious Dehua community atmosphere of daily life, establish the moral high ground, manufactured civilian idols, purify the ecological environment on campus interpersonal communication, to create a good campus cultural exchanges, so that students can understand the sense of belonging and well-being on campus exchanges flu and other positive emotions, recognizing the alienation caused by obstacles and drawbacks, promote body rational exchanges, exchanges overcome utilitarian, the main object of the alienation of.

Second, the use of test functions of daily life, develop a healthy personality and communication ability in daily life, conflict resolution and peer self-identity intimacy. Natural management tools, play the original rational function in daily life, and guide college students to observe life, thinking life, respect for life, and life as a friend, to become master of life. In the world of daily life, recessive means of educating people and procedural details of the influence of education to do the job. Daily life will naturally make a reasonable and appropriate judgment. Needless to say, in a world of alienation exchanges students are confused and depressed, they may boycott the subject of education forced indoctrination obviously didactic, but cannot avoid subtly objective process associated with daily life and change shape.

Third, both the target of daily life, is also a destination. In daily life as the goal, to have the boot sense, as Mr. Tao said: "Education is the good life is good, life is bad is bad education." Educational daily life the most important is that it "has no intention to succeed", subtly so that students lead a moral life on rational, consciously resist the immoral or remote from the alienation of life is the ultimate goal of the daily life education. In daily life as a destination, we do not have high-profile proposed other to get rid of alienation too ambitious a target, as long as able to adapt to the role of daily life and lead a normal and harmonious interpersonal relationships, as evidenced by college students has come out of alienation haze, so more effective sex.

Fourth, pay attention to the overall effect of educating people in daily life. The traditional concept of education is the essence of education and life stripped off, think life is one thing and education is higher than the life of another. Even subjective, some ability and skills will increase with age and the automatic acquisition, college students interpersonal skills belong to this column. Such resources will inevitably lead to inadequate investment in education of the status quo. Daily life educating people adhering to the concept of educating people of all-round development, emphasis on education and training includes basic common quality of all, including the balanced use and distribution of educational resources relating to various aspects of life, educating people to form a joint force from the start with the details of education, investment in education in order to overcome the alienation caused by interaction of alienation remarkable influence.

\section{Conclusion}


From the perspective of daily life, college students should be harmonious interaction forms, they learn to respect other people's personality, master working with people together to complete the task ability, equality between the position of the main contacts back; get rid of the alienation of the media dependence reply Subjective self-interaction plays in the world of daily life in an appropriate and rational protagonist; the conversion from the presence of the original self-conscious to exist; this is the individual students under the guidance of modern education goals overall quality development, and ultimately in line with Marx ideal advocated free comprehensive human development requirements.

\section{REFERENCE:}

[1] Wu Ning, "Criticism of Daily Life-column Fei V Erzhe Thought Research", People's Publishing House, 2007, p. 172.

[2] Xiang Xianming: "Education of Pan - Generalized Pedagogy preliminary exploration", Shanxi Education Press, 2004 edition, p. 280.

[3] "Tao Xingzhi" Volume II, Hunan Education Press, 1985 edition, p. 55. 